Large Hadron Collider Project

\title{
A Possible 1.8 K Refrigeration Cycle for the Large Hadron Collider
}

\author{
F. Millet*, P. Roussel*, L. Tavian ${ }^{* *}$, U. Wagner**
}

\begin{abstract}
The Large Hadron Collider (LHC) under construction at the European Laboratory for Particle Physics, CERN, will make use of superconducting magnets operating below $2.0 \mathrm{~K}$. This requires, for each of the eight future cryogenic installations, an isothermal cooling capacity of up to $2.4 \mathrm{~kW}$ obtained by vaporisation of helium II at $1.6 \mathrm{kPa}$ and $1.8 \mathrm{~K}$. The process design for this cooling duty has to satisfy several demands. It has to be adapted to four already existing as well as to four new refrigerators. It must cover a dynamic range of one to three, and it must to allow continuous pump-down from $4.5 \mathrm{~K}$ to $1.8 \mathrm{~K}$. A possible solution, as presented in this paper, includes a combination of cold centrifugal and warm volumetric compressors. It is characterised by a low thermal load on the refrigerator, and a large range of adaptability to different operation modes. The expected power factor for $1.8 \mathrm{~K}$ cooling is given, and the proposed control strategy is explained.
\end{abstract}

\footnotetext{
* DSM/DRFMC/SBT, CEA Grenoble

F - 38054 Grenoble Cedex 9, France

** CERN - LHC-ACR Division
}

Presented at CEC-ICMC 1997 - Portland OR - USA

July 28th - August 1st, 1997

\author{
Administrative Secretariat \\ LHC Division \\ CERN \\ CH - 1211 Geneva 23 \\ Switzerland
}




\title{
A POSSIBLE $1.8 \mathrm{~K}$ REFRIGERATION CYCLE FOR THE LARGE HADRON COLLIDER
}

\author{
F. Millet, ${ }^{1}$ P. Roussel, ${ }^{1}$ L. Tavian ${ }^{2}$ and U. Wagner ${ }^{2}$ \\ ${ }^{1}$ DSM/DRFMC/SBT, CEA Grenoble \\ F-38054 Grenoble cedex 9, France \\ 2 LHC Division, CERN \\ CH-1211 Geneva 23, Switzerland
}

\begin{abstract}
The Large Hadron Collider (LHC) under construction at the European Laboratory for Particle Physics, CERN, will make use of superconducting magnets operating below $2.0 \mathrm{~K}$. This requires, for each of the eight future cryogenic installations, an isothermal cooling capacity of up to $2.4 \mathrm{~kW}$ obtained by vaporisation of helium II at $1.6 \mathrm{kPa}$ and $1.8 \mathrm{~K}$. The process design for this cooling duty has to satisfy several demands. It has to be adapted to four already existing as well as to four new refrigerators. It must cover a dynamic range of one to three, and it must to allow continuous pump-down from $4.5 \mathrm{~K}$ to $1.8 \mathrm{~K}$. A possible solution, as presented in this paper, includes a combination of cold centrifugal and warm volumetric compressors. It is characterised by a low thermal load on the refrigerator, and a large range of adaptability to different operation modes. The expected power factor for $1.8 \mathrm{~K}$ cooling is given, and the proposed control strategy is explained.
\end{abstract}

\section{INTRODUCTION}

The European Laboratory for Particle Physics, CERN, is working towards the construction of the LHC, a high-energy, high-luminosity particle accelerator and collider of $26.7 \mathrm{~km}$ circumference. The key technology of the LHC is the use of high-field superconducting magnets using $\mathrm{Nb}$-Ti conductor operating in superfluid helium. ${ }^{1}$ To maintain the some $25 \mathrm{~km}$ of bending and focusing magnets at their operating temperature below $2.0 \mathrm{~K}$, the LHC cryogenic system will have to produce an unprecedented total refrigerationcapacity of about $18 \mathrm{~kW}$ at $1.8 \mathrm{~K}$, in eight cryogenic plants distributed around the machine's circumference. To reach this goal, several solutions of process design for the cooling loop of the $1.8 \mathrm{~K}$ refrigeration stage have been studied. 


\section{CONSTRAINTS AND REQUIREMENTS}

The cryogenic system as it will be installed for the LHC machine is facing several constraints. Of the eight refrigerators which are necessary to fulfil the cryogenic capacity demand, four will have to use the already existing installations for the Large Electron Positron collider (LEP). These LEP refrigerators must be upgraded in capacity and completed with a $1.8 \mathrm{~K}$ refrigeration stage.

To limit the number of design solutions, one single type of $1.8 \mathrm{~K}$ refrigeration stage shall be used both to complete the existing $4.5 \mathrm{~K}$ refrigerators of the LEP machine, and for the four new refrigerators. A suitable interface must therefore be found which can be adapted to the existing and to the new $4.5 \mathrm{~K}$ refrigerators.

In view of facility constraints, particularly in underground caverns, the four new $4.5 \mathrm{~K}$ refrigerators will be installed integrally at the surface, ${ }^{2}$ thus differing from the existing LEP units, which are of a split-cold-box design. ${ }^{3}$ To limit the number of cryogenic transfer lines from the tunnel level, where the $1.8 \mathrm{~K}$ refrigeration stage must be located, to the $4.5 \mathrm{~K}$ refrigerators on ground level, it is desirable to connect the $1.8 \mathrm{~K}$ stage via the connections which serve as supply and return between the $4.5 \mathrm{~K}$ refrigerator and the LHC sector.

The demands in refrigeration capacity for the LHC, where all cooling which is to be provided by the $4.5 \mathrm{~K}$ refrigerators is non-isothermal, are totally different from those for LEP, where $80 \%$ of the cooling is done isothermally at $4.5 \mathrm{~K}$. The $1.8 \mathrm{~K}$ refrigeration stage should therefore complete the existing refrigerators in a way that their upgrade for LHC does not require excessive changes at the existing installations.

Due to the strong influence of the operating conditions of the LHC machine on the cryogenic cooling requirements, ${ }^{4}$ the capacity at $1.8 \mathrm{~K}$ will vary by a factor of three between stand-by and full-load operation. The $1.8 \mathrm{~K}$ refrigeration stage must adapt to these variations in cooling capacity. Moreover, each $1.8 \mathrm{~K}$ refrigeration stage must cool down the sector of $3300 \mathrm{~m}$ length from $4.5 \mathrm{~K}$ to below $2.0 \mathrm{~K}$ without running into unstable operation of the cold compressors.

\section{DIFFERENT SOLUTIONS FOR THE $1.8 \mathrm{~K}$ REFRIGERATION STAGE}

A wide range of possible design solutions for the $1.8 \mathrm{~K}$ stage have been studied at CERN and in collaboration with the CEA Grenoble, in order to define a reference solution which satisfies all requirements mentioned in the chapter above. Common to all solutions is the suction pressure of the cold compressors, which is fixed at $1.5 \mathrm{kPa}$. Two principle solutions for the $1.8 \mathrm{~K}$ refrigeration stage were identified :

a) cycles withoutambient-temperature compressors

b) cycles with ambient-temperature compressors

Figure 1 shows the principle block diagrams for these two solutions. Cycles including ambient-temperature compressors can further be "active" i.e. expand high-pressure helium in turbo expanders and thus create cooling capacity. In both cases, the cold compressor box (CCB) receives helium at $4.5 \mathrm{~K}$ from the main cold box, supplies the $1.8 \mathrm{~K}$ cooling loop, and feeds helium back at a certain temperature level to the main cold box. 

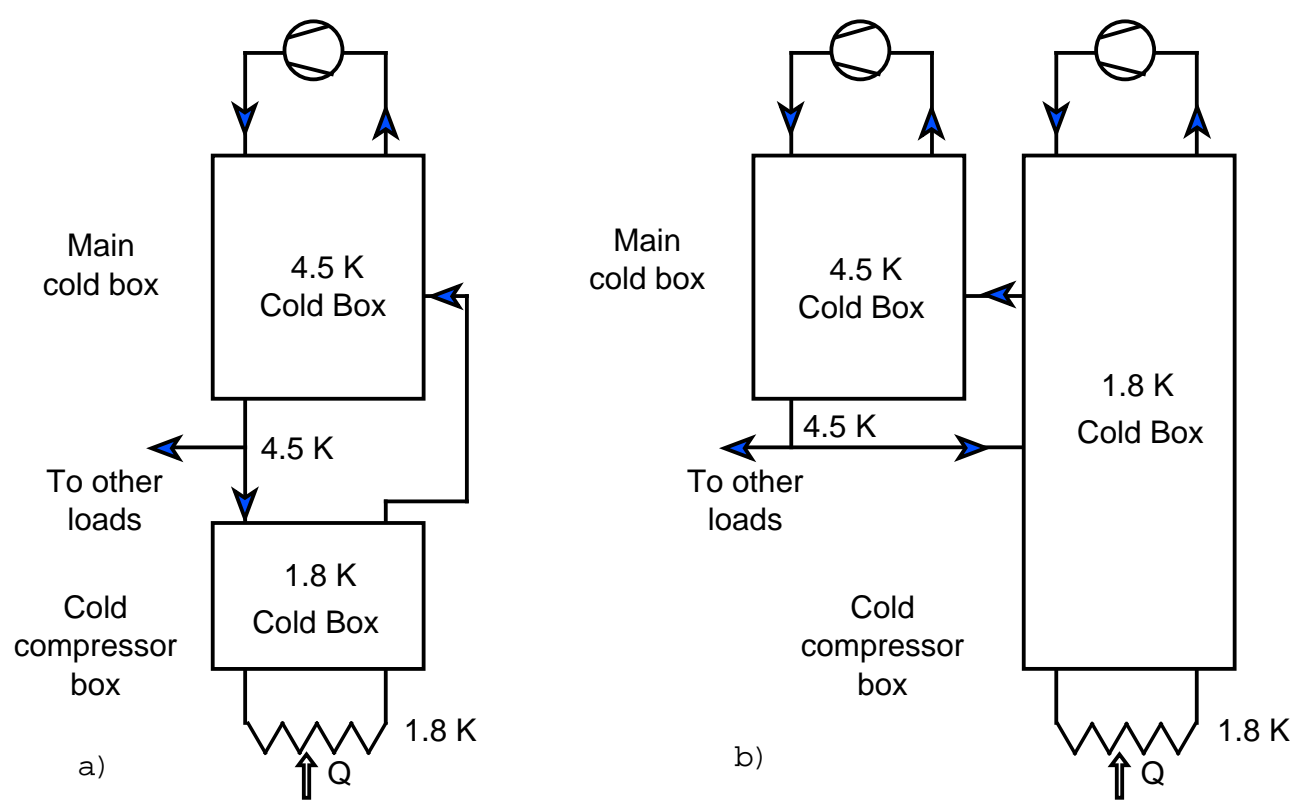

Figure 1. The principle choice for the $1.8 \mathrm{~K}$ refrigeration stage; a) cycles without ambient-temperature compressors, b) cycles with ambient-temperature compressors

\section{Possible Interface Conditions}

The possible interface conditions between the CCB and the main cold box, which apply equally to the existing and to the new $4.5 \mathrm{~K}$ refrigerators, are listed in Table 1.

Table 1. Possible interface conditions between CCB and main cold box

\begin{tabular}{lcc}
\hline & Temperature $[\mathrm{K}]$ & Pressure [MPa] \\
\hline Supply & 4.5 & 0.30 \\
Return A & 20 & 0.13 \\
Return B & 50 & 1.85 \\
Return C & 75 & 1.60 \\
\hline
\end{tabular}

\section{Cold Compressor Cycles without Ambient-Temperature Compressors}

Cold compression to more than $0.13 \mathrm{MPa}$ is excluded by the design of the cold hydrodynamic compressors as well as by the excessive exergetic losses it would create. For solutions without ambient-temperaturecompressor stages, the only possible return interface is at $20 \mathrm{~K}$ and $0.13 \mathrm{MPa}$ as shown in Figure 2. A comparable cycle was realised for the CEBAF refrigerator system. ${ }^{5}$ Unlike the CEBAF example, these solutions would need additional cooling capacity to be provided by the $4.5 \mathrm{~K}$ refrigerator in order to reach the $20 \mathrm{~K}$ limit, as cold compression from $1.5 \mathrm{kPa}$ to $0.13 \mathrm{MPa}$ leads to discharge temperatures between $40 \mathrm{~K}$ and $50 \mathrm{~K}$ for compressors with an isentropic efficiency between $75 \%$ and $60 \%$, respectively. All CCB cycles without ambient compressors therefore increase the capacity demands on the main cold box, which is particularly undesirable for the existing installations.

\section{Cold Compressor Cycles with Ambient-Temperature Compressors}

Cold compression with ambient-temperature compressors could have any return interface at temperature levels between $300 \mathrm{~K}$ and $4.5 \mathrm{~K}$, and pressure levels up to the supply value from the ambient-temperature compressors. 


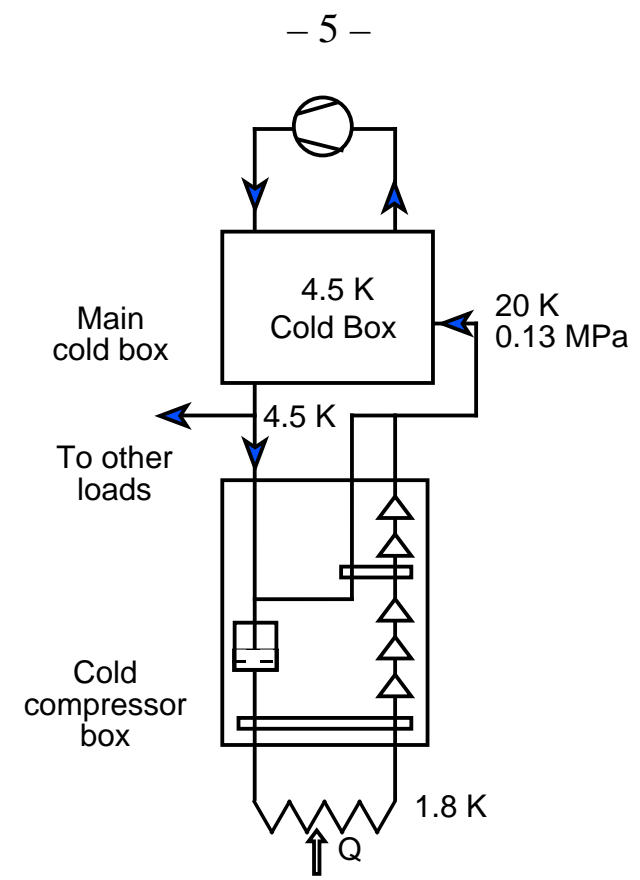

Figure 2. The $1.8 \mathrm{~K}$ refrigeration stage without ambient-compressors connected to a $4.5 \mathrm{~K}$ refrigerator via the possible interface connections (Return A)
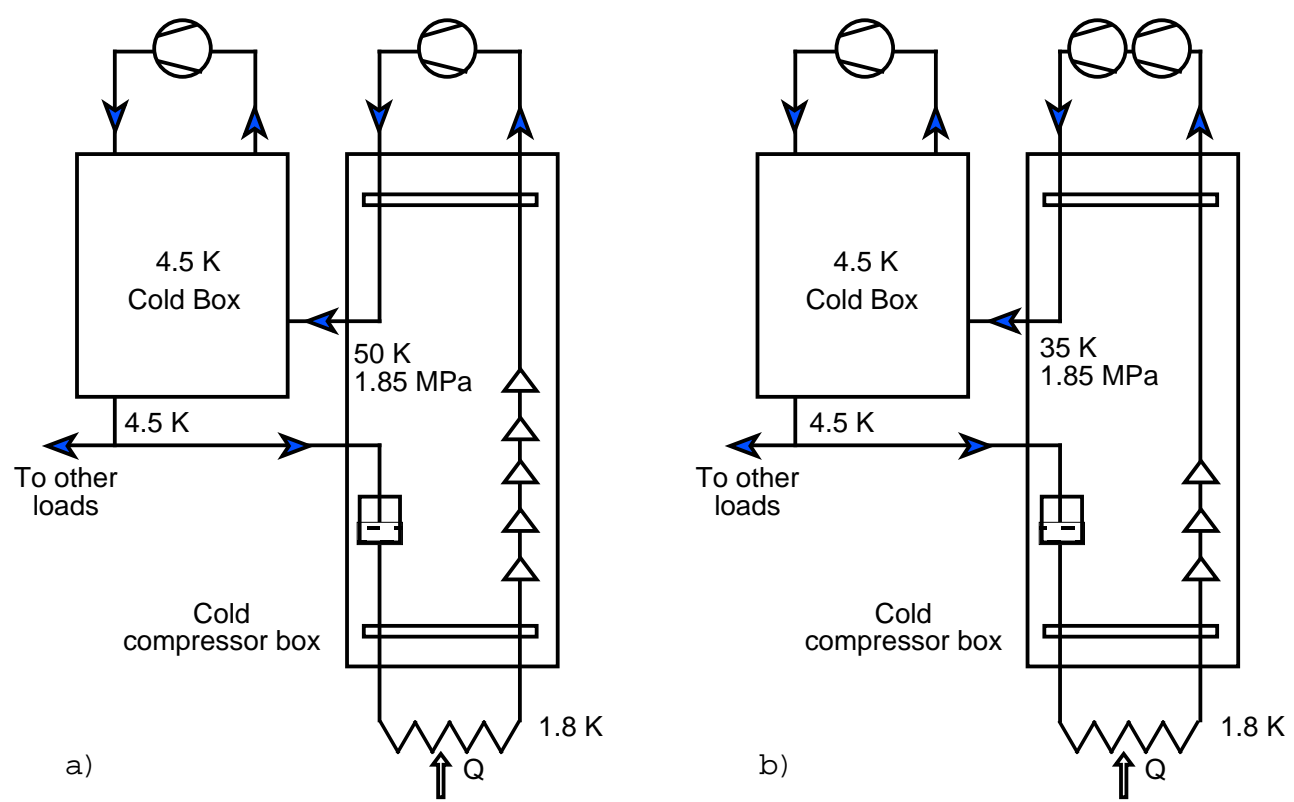

Figure 3. $1.8 \mathrm{~K}$ refrigeration stages including ambient-temperature compressors with no creation of cooling capacity via expanders; a) with cold compression to pressures above $0.1 \mathrm{MPa}, \mathrm{b}$ ) with cold compression to pressures below $0.1 \mathrm{MPa}$.

The block diagrams of four different solutions including compression at ambient temperature are shown in Figure 3 and Figure 4. The two cycles in Figure 3 are characterised by the fact that no cooling capacity is created by expansion of helium gas in the cold compressor box. The helium returned to the main cold box at high pressure is expanded in turbines located therein. The difference between the two cycles in Figure 3 is that in one case five stages of cold centrifugal compressors pump the helium from $1.5 \mathrm{kPa}$ to above atmospheric pressure, whereas in the other case cold compression is only used to a pressure below atmospheric level, thus requiring warm machines with sub-atmospheric suction. A cycle with two stages of cold centrifugal compressors and sub-atmospheric suction was already realised for the refrigerator of the Tore Supra superconducting tokamak. ${ }^{6}$ 

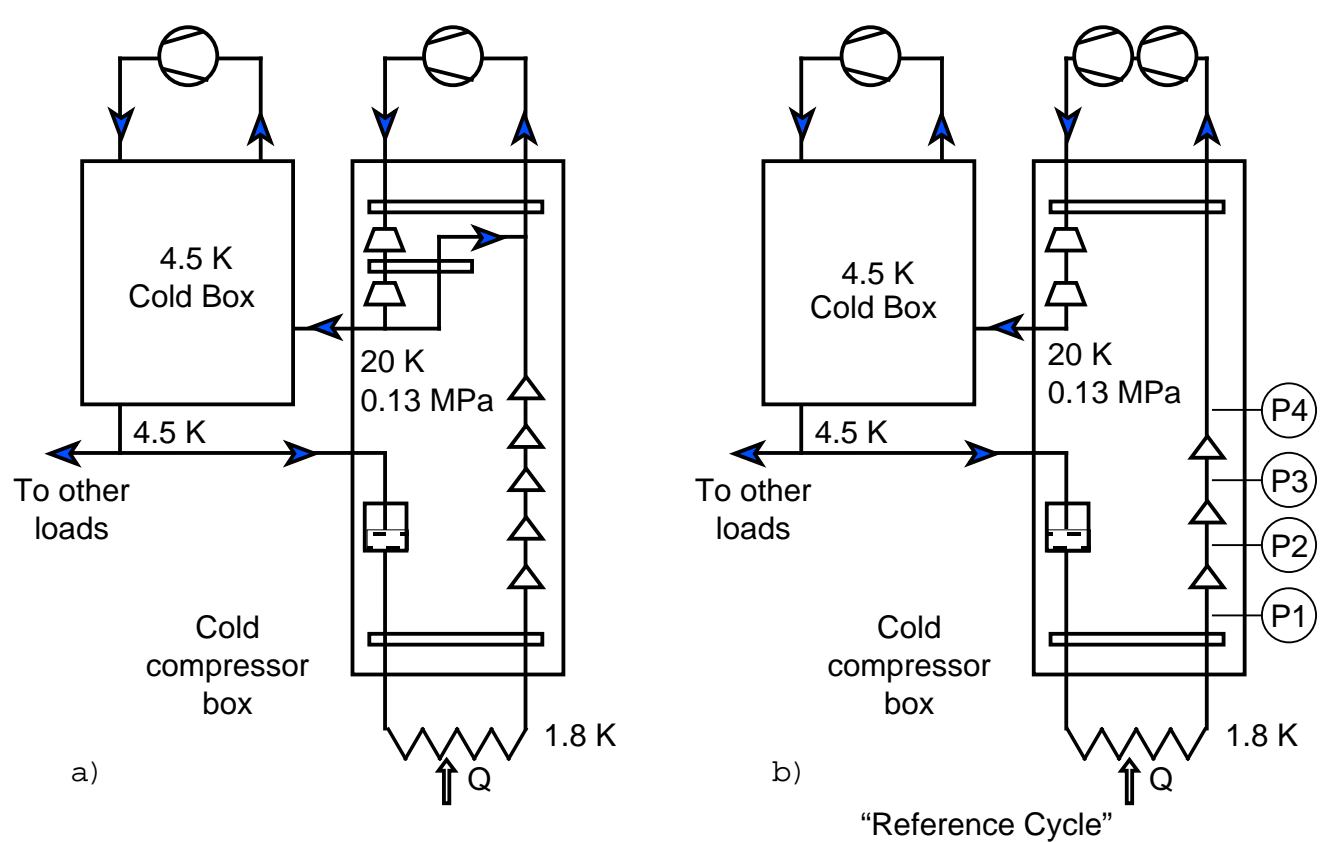

Figure 4. $1.8 \mathrm{~K}$ refrigeration stages including ambient-temperature compressors with creation of cooling capacity in turbo expanders; a) with cold compression to pressures above $0.1 \mathrm{MPa}, \mathrm{b}$ ) with cold compression to pressures below $0.1 \mathrm{MPa}$.

The two cycles in Figure 4 are characterised by the fact that the high-pressure helium is expanded in turbines inside the cold compressor boxes. The helium gas is in both cases returned to the main cold box at $20 \mathrm{~K}$ and $0.13 \mathrm{MPa}$.

\section{THE 1.8 K CYCLE PROPOSED BY CERN AS REFERENCE SOLUTION}

The cycle which is represented on the right-hand side of Figure 4 was chosen by CERN as a suitable reference for comparison with other solutions, after evaluating its possible advantages and disadvantages. It uses three stages of cold centrifugal compressors, with a pressure ratio of three for each stage, up to a pressure level of about $40 \mathrm{kPa}$. At this pressure, efficient heat exchange in standard aluminium-plate-fin heat exchangers is possible. For ambient-temperature compressors, either a combination of liquid ring pumps up to 0.11 $\mathrm{MPa}$ and helical screw compressors for the high pressure level, or only helical screw compressors can be chosen. The overall efficiency of this cycle depends strongly on the efficiency of the warm machinery, which would favour the use of screw compressors. Even with isentropic efficiencies of $60 \%$ for the cold compressors, which is considered as conservative for the size of compressors to be installed for the LHC, a return temperature of $20 \mathrm{~K}$ or lower can be reached without additional cooling flow inside the CCB. During reduced capacity operation, the suction pressure of the warm volumetric compressors can be left floating, which eases considerably the control of the centrifugal machines and expands the operation range of the whole cycle. Moreover, this cycle contributes to the total refrigeration capacity, which decreases the demand on the main cold boxes. This is specially of interest for the reuse of the existing LEP refrigerators. 


\section{Advantages of the Reference Solution}

The advantages of a CCB cycle with a mixture of cold centrifugal and warm volumetric compression are:

- good cycle efficiency,

- lowering of the capacity demand for the main refrigerator,

- good adaptability to reduced-capacity operation,

- good controllability during cool-down of a machine sector below $4.5 \mathrm{~K}$.

\section{Disadvantages of the Reference Solution}

The only disadvantage of the $1.8 \mathrm{~K}$ cycle proposed as reference solution is that the first stage of the warm volumetric compressors has a sub-atmospheric suction pressure. This is generally avoided in helium refrigerators as it increases the risk of air contamination in the process gas. The experience of the French CEA with the Tore Supra refrigerator shows however, that a carefully designed warm sub-atmospheric system allows reliable operation. Moreover, a cryogenic system the size of one LHC sector has a multitude of inherent sources for contamination. An efficient purification system has to be installed, which can also be designed to cope with possible impurities resulting from the sub-atmospheric suction of the warm compressors.

\section{REDUCED-CAPACITY OPERATION}

The $1.8 \mathrm{~K}$ cycle must be capable of operating continuously with flow rates down to a third of its nominal value. As operation at low flow can occur for extended time periods, it is not desirable to compensate by electrical heating in a $1.8 \mathrm{~K}$ bath. ${ }^{7}$ Hydrodynamic cold compressors cannot easily match this flow variation at constant pressure ratio. In the reference solution for the $1.8 \mathrm{~K}$ cycle, it is envisaged to let the suction pressure of the warm volumetric machines decrease proportional with the decrease of flow, from e.g. $40 \mathrm{kPa}$ at full load to $13 \mathrm{kPa}$ at low capacity. A feasible and rather simple control strategy for three cold compressors in series is given in Table 2. The identifiers for the pressure points refer to those shown in Figure 4. The lowest-pressure stage is controlled to a fixed suction pressure of 1.5 $\mathrm{kPa}$. The second stage is controlled to have a third of the total pressure ratio between suction of the first stage and discharge of the third stage. The third stage is controlled to a fixed pressure ratio of three in all load cases. The control is done for each cold compressor by varying the rotational speed. Following this control strategy, all centrifugal compressors stay well within their possible field of operation with a comfortable distance from the stall and choke limits.

Table 2. Control strategy for the cold compressors of the reference cycle during turn-down operation

\begin{tabular}{lll}
\hline Cold compressor & Control strategy & Control variable \\
\hline First stage & $\mathrm{P} 1=1.5 \mathrm{kPa}$ & Rotational speed first stage \\
Second stage & $\mathrm{P} 3 / \mathrm{P} 2=(\mathrm{P} 4 / \mathrm{P} 1)^{1 / 3}$ & Rotational speed second stage \\
Third stage & $\mathrm{P} 4 / \mathrm{P} 3=3$ & Rotational speed third stage \\
\hline
\end{tabular}




\section{POWER FACTOR}

To calculate the expected power factor for $1.8 \mathrm{~K}$ cooling, assumptions must be made concerning the efficiency of cold compressors, heat exchangers, turbo expanders and warm volumetric compressors, as well as of the future $4.5 \mathrm{~K}$ refrigerators.

For the cold centrifugal compressors, an isentropic efficiency of $60 \%$ seems reasonable, while that estimated for the turbo expanders is $70 \%$. The heat exchanger efficiency is assumed to be $96 \%$. For warm screw compressors with sub-atmospheric suction, an isothermal efficiency of $30 \%$ could be envisaged; for screw compressors operation above $0.1 \mathrm{MPa}$ suction this value can be assumed to $50 \%$. The Carnot efficiency of the cold box for the main refrigerator is further estimated to be $50 \%$.

Using these rather conservative efficiency figures, the power factor for the $1.8 \mathrm{~K}$ cooling would be about $1060 \mathrm{~W} / \mathrm{W}$ in the case of the proposed reference cycle. If for comparison one uses the same assumptions to calculate the power factor of a cycle as it is represented in Figure 4 a, the resulting value would be 1170 W/W.

\section{CONCLUSION}

The cold compressor cycle which is proposed by CERN to be combined with the $4.5 \mathrm{~K}$ cold boxes of the LHC cryogenic system, is adaptable to the existing refrigerators of the LEP machine, which are of split design, as well as to the all surface installed new $4.5 \mathrm{~K}$ refrigerators, which will have to be installed in the coming years. No additional cryogenic transfer line connections are necessary besides those already foreseen to supply the LHC machine from the $4.5 \mathrm{~K}$ refrigerators. The cycle allows reduced-capacity operation to a third of the nominal mass flow, and continuous pump-down of the LHC sector from $4.5 \mathrm{~K}$. Moreover, it shows a promising potential for good overall efficiency. The sub-atmospheric suction of its warm compressors demands careful design and provisions to remove air contamination from the process helium.

\section{ACKNOWLEDGEMENTS}

This work was performed in the framework of a collaboration agreement between CERN and CEA, Grenoble and Cadarache, France.

1. The LHC study group, "The Large Hadron Collider, Conceptual Design," CERN Report/AC/95-05(LHC)

2. U. Wagner, "The LHC refrigerators with surface-locatedcold boxes for the temperature range 300-4.5 K," LHC Project Note 70 (1996)

3. S. Claudet, W.K. Erdt, P.K. Frandsen, Ph. Gayet, N.O. Solheim, Ch. Titcomb and G. Winkler, Four 12 kW / 4.5 K cryoplants at CERN, Cryogenics (1994) 34 ICEC Supplement, p. 83-86

4. Ph. Lebrun, G. Riddone, L. Tavian and U. Wagner, Demands in refrigeration capacity for the Large Hadron Collider, in: "Proceedings of ICEC 16," T. Haruyama, T. Mitsui, K. Yamafuji, ed., Elsevier Science (1997), p. 95-98

5.C.H. Rode, D, Arenius, W.C. Chronis, D. Kashey and M. Keesee, 2.0 K CEBAF cryogenics, in: “Advances in Cryogenic Engineering," R.W. Fast, ed. Plenum Press, 35A (1990), p. 275-286

6. G. Claudet and R. Aymar, Tore supra and helium-II cooling of large high-field magnets, in: "Advances in Cryogenic Engineering," R.W. Fast, ed. Plenum Press, 35A (1990), p. 55-67

7.B.S. Bevins, W.C. Chronis, andM.S. Keesee, Automatic pumpdown of the $2 \mathrm{~K}$ cold compressors for the CEBAF central helium liquefier, Advances in Cryogenic Engineering Vol 41, Plenum Press, N.Y. (1996), p.663. 